\title{
Article \\ Influence of Retinal Microsecond Pulse Laser Treatment in Central Serous Chorioretinopathy: A Short-Term Optical Coherence Tomography Angiography Study
}

\author{
Michelle Prasuhn ${ }^{1,2, *,+} \mathbb{D}$, Yoko Miura ${ }^{1,3, *,+} \mathbb{C}$, Aysegül Tura ${ }^{1}$, Felix Rommel ${ }^{1,2} \mathbb{D}$, Vinodh Kakkassery ${ }^{1} \mathbb{D}$, \\ Svenja Sonntag ${ }^{1}$, Salvatore Grisanti ${ }^{1}$ and Mahdy Ranjbar ${ }^{1,2}$ \\ 1 Department of Ophthalmology, University Hospital Schleswig-Holstein, University of Lübeck, \\ Ratzeburger Allee 160, 23538 Lübeck, Germany; Ayseguel.Tura@uksh.de (A.T.); felix.rommel@uksh.de (F.R.); \\ Vinodh.Kakkassery@uksh.de (V.K.); svenja.sonntag@uksh.de (S.S.); salvatore.grisanti@uksh.de (S.G.); \\ eye.research101@gmail.com (M.R.) \\ 2 Laboratory for Angiogenesis \& Ocular Cell Transplantation, University Hospital Schleswig-Holstein, \\ University of Lübeck, Ratzeburger Allee 160, 23538 Lübeck, Germany \\ 3 Institute of Biomedical Optics, University of Lübeck, Peter-Monnik-Weg 4, 23562 Lübeck, Germany \\ * Correspondence: michelle.prasuhn@uksh.de (M.P.); yoko.miura@uni-luebeck.de (Y.M.); \\ Tel.: +49-451-3101-3212 (Y.M.) \\ $\dagger$ These authors contributed equally to this work.
}

Citation: Prasuhn, M.; Miura, Y.; Tura, A.; Rommel, F.; Kakkassery, V.; Sonntag, S.; Grisanti, S.; Ranjbar, M. Influence of Retinal Microsecond Pulse Laser Treatment in Central Serous Chorioretinopathy: A Short-Term Optical Coherence Tomography Angiography Study. J. Clin. Med. 2021, 10, 2418. https:// doi.org/10.3390/jcm10112418

Academic Editors:

Andrzej Grzybowski and

Emmanuel Andrès

Received: 5 May 2021

Accepted: 27 May 2021

Published: 29 May 2021

Publisher's Note: MDPI stays neutral with regard to jurisdictional claims in published maps and institutional affiliations.

Copyright: (c) 2021 by the authors. Licensee MDPI, Basel, Switzerland. This article is an open access article distributed under the terms and conditions of the Creative Commons Attribution (CC BY) license (https:// creativecommons.org/licenses/by/ $4.0 /)$.
Abstract: Background: Central serous chorioretinopathy (CSC) is a common macular condition characterized by detachment of the neuroretina and is a frequent cause of central vision loss in adults. Among the various therapeutic strategies, subthreshold microsecond pulsed laser (SML) treatment has become a useful option. Despite the suggested involvement of choroidal circulatory disturbances in CSC, the effects of this treatment on macular microperfusion have not been fully evaluated yet. Herein, we report the impact of SML on retinal and choroidal microvascular flow using non-invasive optical coherence tomography (OCT) angiography (OCTA). Methods: In this study, CSC patients with persistent subretinal fluid (SRF) with or without secondary choroidal neovascularization (CNV) were included (referred to as the pachychoroid neovasculopathy (PNV) group and the CSC group, respectively). SML was conducted using a yellow $(577 \mathrm{~nm})$ laser with a duty cycle of $10 \%$, spot size of $200 \mu \mathrm{m}$ and duration of $200 \mathrm{~ms}$. Best corrected visual acuity (BCVA) as well as OCT and OCTA images were evaluated at baseline and 4 weeks after SML. OCTA parameters of interest included full retinal perfusion (FRP), choriocapillaris perfusion (CCP), Sattler's layer perfusion (SLP), and Haller's layer perfusion (HLP), which were evaluated longitudinally and compared to unaffected fellow eyes. Results: 27 affected eyes and 17 fellow eyes from 27 patients were included. Before treatment, central retinal thickness (CRT) and subfoveal choroidal thickness (SFCT) of affected eyes were significantly larger than in fellow eyes. Four weeks after SML, CRT decreased significantly, whereas perfusion parameters did not change. In subgroup analyses, the CSC group showed a significant decrease in SFCT, whereas the PNV group did not despite the decrease in CRT. Conclusion: Our results suggest that the SML may affect the SFCT of the CSC, but not the PNV patients at least within four weeks following treatment. This effect seems to be independent of the change in choroidal perfusion measured with OCTA.

Keywords: OCTA; central serous chorioretinopathy; choroidal perfusion; choriocapillaris; Sattler's layer; Haller's layer; retinal perfusion; subthreshold micropulse laser

\section{Introduction}

Central serous chorioretinopathy (CSC) is a common chorioretinal disease and a frequent cause of central vision loss, primarily affecting people between 20 to 60 years of age. It is characterized by serous detachment of the neuroretina, typically with localized 
disruption of the outer blood retinal barrier, where the tight junctions of the retinal pigment epithelium (RPE) are localized [1]. CSC is an entity of the pachychoroid disease spectrum, in which the choroidal vessels are typically dilated, and leakage might be seen in indocyanine green angiography (ICGA) [2,3]. This mechanism is suggested to lead to choriocapillaris hypoperfusion, consequently causing localized RPE damage [4]. Conditions of the pachychoroid disease spectrum are characterized by a focal or diffuse increase in choroidal thickness. Specific findings include dilated vessels in the Haller's layer, the so called pachyvessels. The choroidal substructures are altered and a shift in thickness as well as perfusion from the choriocapillaris (CC) and Sattler's layer (SL) towards Haller's layer (HL) can be seen [5]. CSC patients may develop choroidal neovascularizations (CNVs), which have recently been termed pachychoroid neovasculopathy $(\mathrm{PNV})$ and is also part of the pachychoroid disease spectrum [6].

Patients often complain of substantial visual impairment, which consequently affects their quality of life. Treatment for CSC aims to preserve outer sensory retina layers and achieve quick and complete resolution of SRF. Because of the frequently self-limiting course, treatment modalities should have a favorable safety profile.

Photocoagulation at RPE leakage points is a traditional evidence-based method to treat CSC. However, it has several serious drawbacks, including symptomatic scotomas, foveal distortion, and subretinal fibrosis, and thus has to be avoided in cases with subfoveal to juxtafoveal leaking points [7]. Enlargement of the laser spot may also threaten central vision. In the last decades, further treatment options have been introduced: diuretics, half-dose photodynamic therapy (PDT), minimally-invasive laser therapy such as selective retina therapy (SRT), and treatment with a microsecond pulsed laser. One of the diuretics, eplerenone, is a steroidal antimineralocorticoid and has been used widely with CSC patients in recent years [8]. Although its potential effect on the choroidal blood flow has been suggested [9], recent study results showed no significant therapeutic effect of eplerenone in chronic CSC patients $[10,11]$. Half-dose PDT is a safe and effective method to treat juxtafoveal or subfoveal leakage [9]. However, long-term adverse effects or cumulative effects of repeated treatments, such as RPE atrophy, still cannot be excluded and thus caution should be exerted [12]

Subthreshold microsecond pulsed laser (SML) treatment has also been proposed as a new therapeutic strategy in CSC. This is a method to reduce the total energy of the irradiated laser pulse by reducing the on-time during one irradiation. It may easily achieve a very mild increase in temperature of RPE cells such that cells are sublethally heated or just mildly coagulated. Sublethal RPE hyperthermia has been shown to activate different intracellular molecules, such as heat shock protein 70 [13-15] and water transport protein aquaporine [16]. Furthermore, it has been shown to increase the antioxidative potential $[17,18]$ and to reduce the thickness of the Bruch's membrane in an age-related macular degeneration (AMD) mice model [19-22]. Thus, sublethal RPE thermal treatment gained increasing attention as a new therapeutic option for different macular disorders, such as CSC, diabetic macular edema and AMD [20,23]. In particular, the PLACE trial and a recent meta-analysis demonstrated that SML may be considered as a competitive alternative to PDT for treating chronic CSC, and as the first-line treatment of chronic CSC $[24,25]$.

Although there is increasing clinical evidence for the treatment of CSC using SML [20], the effect of the treatment on the choroid has not been thoroughly investigated to date. Therefore, in the present study, we aimed to investigate structural and functional alterations of the retina and the choroidal sublayers in patients with CSC before and after SML using OCT and OCTA.

\section{Materials and Methods}

\subsection{Study Participants}

The participants for this prospective observational study were enrolled at the Department of Ophthalmology of the University of Lübeck. The study was positively reviewed 
by the institutional review board and adhered to the tenets of the Declaration of Helsinki (ID 18-102). All participants received detailed information about the study, and written informed consent was obtained individually by each participant before enrolment. We included patients who were diagnosed with CSC based on the SRF involving the macula as demonstrated by spectral domain optic coherence tomography (SD-OCT) and persistent SRF for at least 3 months. Both local and diffuse leakage on FA were included in this study. Exclusion criteria were: (1) history of any other macular disease; (2) any laser treatment within six months before enrolment (3); intravitreal injection within three months of enrolment; (4) large RPE atrophy; (5) history of ocular diseases other than CSC in the fellow eye; (6) previous PDT. Patients who were treated with diuretics, such as acetazolamide and eplerenone, were included in the study. As control, we examined the fellow eyes, which received the same multimodal imaging modalities. If the fellow eyes had a history of previous retinal disease, including active or inactive CSC, or showed signs of CSC in ICGA, OCTA, or OCT these eyes were excluded from the analysis. For subgroup analyses, we differentiated between CSC patients without CNV and PNV patients. The latter was characterized by the presence of a CNV, which was diagnosed using FA, OCT, ICGA, and OCTA by two experienced ophthalmologists in a consensus process (Y.M. and M.P.).

\subsection{Study Protocol}

At baseline, all participants underwent a thorough examination including refraction, best-corrected visual acuity (BCVA) in Snellen, intraocular pressure (IOP), slit-lamp biomicroscopy, macular enhanced-depth imaging (EDI) OCT, FA, ICGA, FAF as well as OCTA. The maximum permissible spherical and cylindrical aberration was \pm 3 and \pm 1 diopters, respectively. Imaging was performed using the Zeiss Cirrus HD-OCT (AngioPlex, CIRRUS HD-OCT model 5000, Carl Zeiss Meditec, Inc., Dublin, CA, USA) by a single, trained operator. Each imaging session included EDI-OCT $\left(10 \times 10 \mathrm{~mm}^{2}\right)$ and OCTA $\left(6 \times 6 \mathrm{~mm}^{2}\right)$ volumetric scans of the posterior pole. Only scans with a signal strength $\geq 7$, centered on the fovea, and without motion-, segmentation-, and projection artifacts were included [26]. To avoid inaccuracies due to diurnal changes in perfusion, OCTA images were acquired at the same $2 \mathrm{~h}$ time frame of the day $[27,28]$. One month after treatment, all participants underwent the same examination of both eyes, however without FA.

\subsection{Laser Treatment}

Laser irradiation was conducted using a yellow laser $(577 \mathrm{~nm})$ with a system for microsecond pulsing (MicroPulse TxCell Scanning Laser Delivery System; Iridex, Mountain View, CA, USA or Truscan; LIGHTMED, San Clemente, CA, USA), with a spot size of $200 \mu \mathrm{m}$ and a duration of $200 \mathrm{~ms}$ with a $10 \%$ duty cycle. Test irradiation was conducted outside of the upper or lower vascular arcade, starting from a power of $200 \mathrm{~mW}$, with increments of $100 \mathrm{~mW}$, until a mild white spot was observed (determination of the visibility threshold). Treatment was conducted with the power of $40-50 \%$ of the visibility threshold around the leakage points observed in FA. Irradiation was conducted with a spacing about 1 spot diameter. In cases where there was no leakage point or diffuse leakage, SML was applied diffusely at the area of SRF.

\subsection{OCT and OCTA Data Analysis}

Central retinal thickness (CRT) was automatically measured by the device, as defined by the Early Treatment Diabetic Retinopathy Study (ETDRS) in the OCT scans [29]. The total macular volume (TMV) was also automatically calculated. Subfoveal choroidal thickness (SFCT) was measured manually in EDI-OCT scans just below the fovea, extending perpendicularly from the hyperreflective Bruch's membrane layer to the inner scleral border.

Figure 1 shows an overview of OCTA segmentation. Figure 2 visualizes the OCT analyses to characterize the extent of the SRF. The maximum point of the SRF was determined and the focal retinal thickness was automatically measured using the ETDRS grid (lesional 
retinal thickness $=$ LRT). Additionally, in the B-scan with the maximum SRF, the area of SRF was measured using the device's measuring tool (apical SRF $=$ aSRF). We also assessed the SRF base area, which was pictured in the inner segment/outer segment images of the device and was calculated using the area measuring tool in ImageJ ((NIH, Version 1.48b, Bethesda, MD, USA). Manual measurements were performed by two experienced graders (F.R. and M.P.) who were blinded to the clinical information about the examined eyes and the results were averaged.

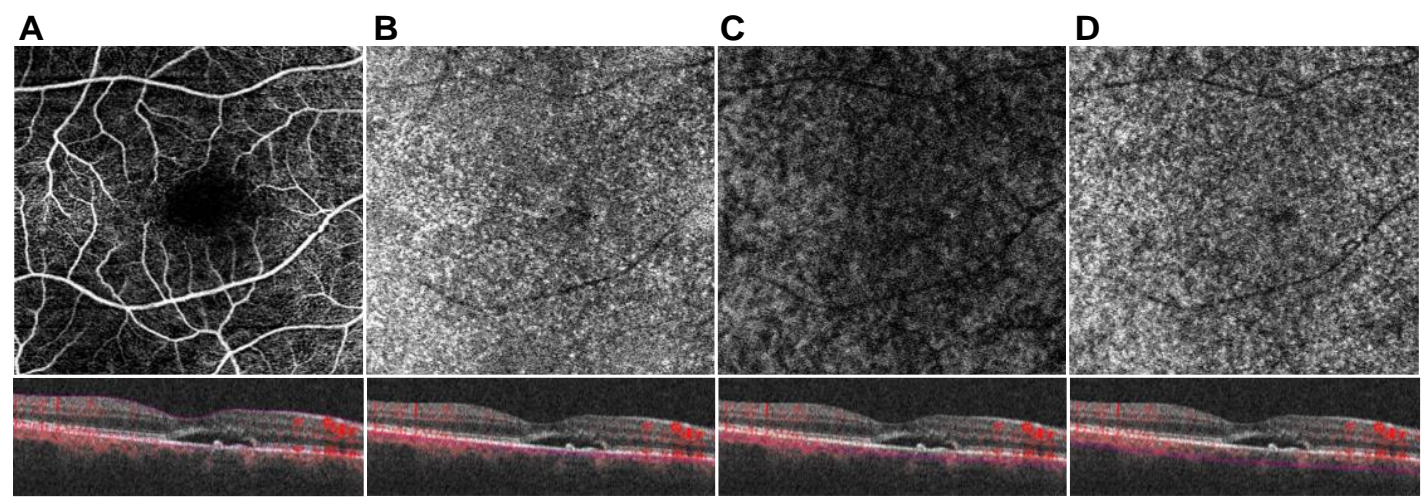

Figure 1. Optical coherence tomography angiography (OCTA)-Imaging of the posterior pole in a subject with Central Serous Chorioretinopathy. En face angiogram and corresponding B-scan at the level of the full retina (A), choriocapillaris (B), Sattler's layer (C), and Haller's layer (D).
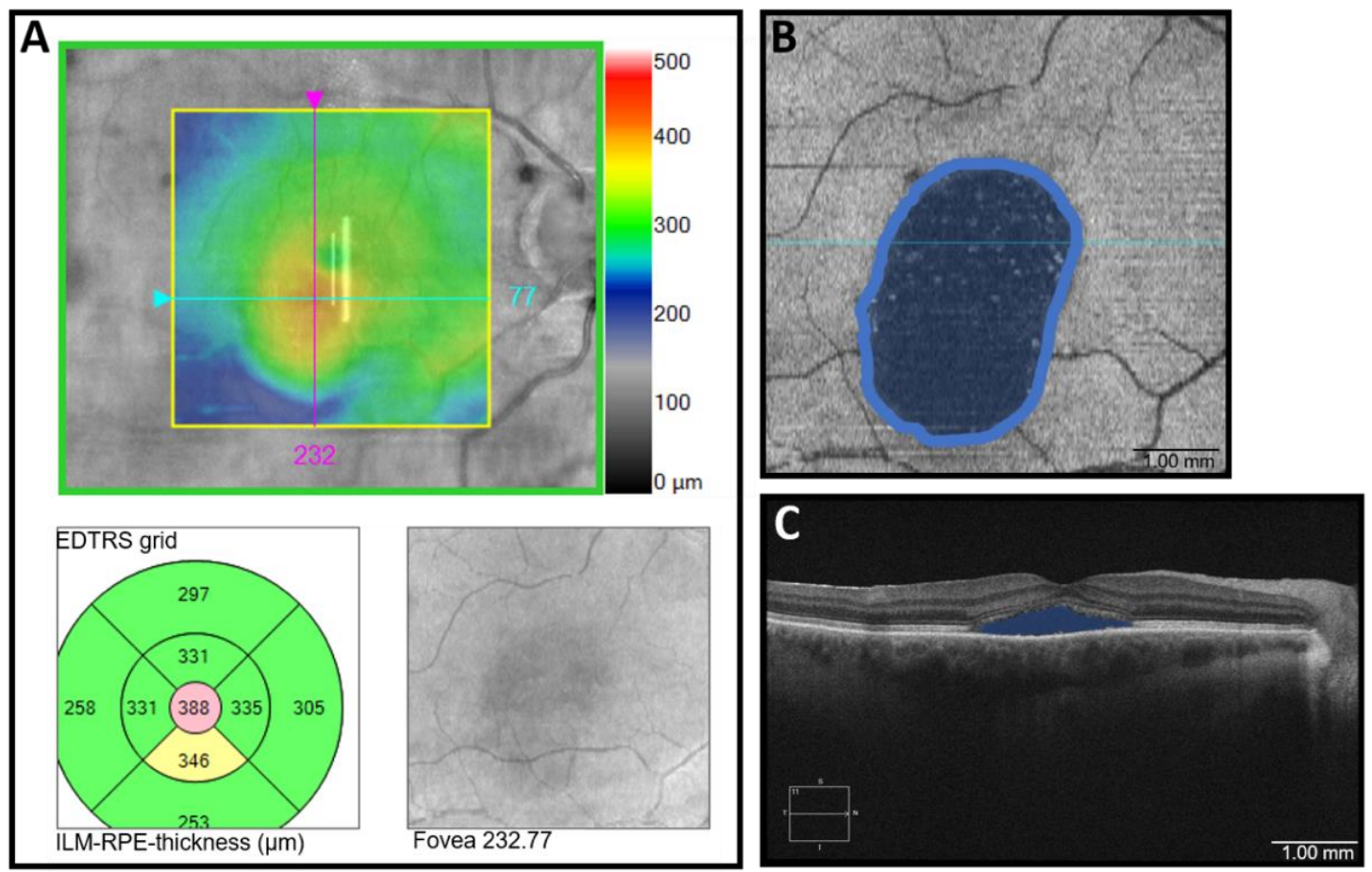

Figure 2. Visualization of methods in optical coherence tomography (OCT) and OCT- angiography in a patient with Central Serous Chorioretinopathy (A): Determining lesional retinal thickness = LRT: En face Analysis. The top panel shows an image of a retinal thickness map. The thickest point with the maximum extent of subretinal fluid (SRF) was determined and fixed, and the EDTRS grid was centered on this point (bottom left panel) to determine the retinal thickness (388 $\mu \mathrm{m})$. Inner limiting membrane = ILM; Retinal pigment epithelium = RPE. (B): Measuring SRF area: Inner segment/outer segment ellipsoid analysis pictures of the base area of the SRF spot. The area was measured in ImageJ. (C): Measuring apical SRF = aSRF: OCT B-scan. The maximum extent of SRF was measured using the device's measuring tool. 
OCTA data were manually segmented in all B-Scans to achieve retinal and choroidal sublayer slabs. Slabs for full retina perfusion (FRP) were measured from the Bruch's membrane to the inner limiting membrane (ILM). The choroidal sublayers were manually segmented to get representative $20 \mu \mathrm{m}$ slabs of the CC, SL, and HL according to previously published protocols [26,27]. All acquired en face images were exported into ImageJ for thresholding. Binarization was performed using the Otsu method, which is an automatic threshold selection from grey-level histograms [30]. As suggested by Nicolò et al., CC perfusion (CCP) was calculated by scoring the percentage of white pixels, while for SL perfusion (SLP) and HL perfusion (HLP) the values of black pixels were taken into account [31]. As previously mentioned, a feature of pachychoroidal diseases is a perfusion shift from CC and SL towards HL. To calculate the specific shift of perfusion between the choroidal sublayers, ratios between CCP, SLP, and HLP were determined (CCP/HLP, and SLP/HLP). To analyze the perfusion metrics in the pathological area, a circle of $1000 \mu \mathrm{m}$ diameter was set around the maximum extent of SRF and perfusion values were calculated for CC, SL, HL, FR as described above using the Otsu method for binarization (=lesional CCP, lesional HLP, lesional SLP). The specific perfusion shifts were also calculated below the encircled lesion (=lesional CCP/HLP and lesional SLP/HLP) as described above.

\subsection{Statistical Analysis}

Exploratory data analysis was calculated using IBM SPSS (Version 24.0, Chicago, IL, USA) and figures were generated using Prism GraphPad (Version 8.0, La Jolla, CA, USA). Snellen BCVA was converted to the logarithm of the minimum angle of resolution $(\log$ MAR). Quantitative variables were summarized as median and Quartiles (Q1 and Q3) and qualitative variables as frequency and percentage. The Shapiro-Wilk test was used to check for normality of the obtained data. Baseline and follow-up values of the same eye were compared using a Wilcoxon signed rank test. Differences between CSC eyes and fellow eyes were assessed by a Mann-Whitney U test. For all tests, values of $p<0.05$ were considered statistically significant.

\section{Results}

Twenty-seven CSC patients were considered eligible and were included in this study. Seventeen unaffected fellow eyes served as a control. The demographic features of these groups are listed in Table 1. The anterior segment examinations did not reveal any relevant features. The biomicroscopic fundus examinations revealed serous retinal detachment at the posterior pole, particularly in the macular region, and multiple zones of RPE alterations (hypo- as well as hyper-pigmentations and atrophic changes). Neither drusen nor subretinal hemorrhages were detected.

Table 2 summarizes the anatomical and functional parameters of the CSC and fellow eyes. Eyes with CSC showed a significantly worse BCVA with an increased CRT and SFCT. Perfusion values of the retina and choroidal sublayers did not differ significantly.

Anatomical and functional parameters at baseline and follow-up are presented in Table 3. CRT decreased significantly after SML (from $306 \mu \mathrm{m}$ to $266 \mu \mathrm{s}, p<0.05$ ). The median value of the LRT decreased significantly after SML. We did not detect any significant changes in other parameters, including BCVA, SFCT, SRF and perfusion values.

Table 1. Demographic and clinical characteristics of enrolled CSC patients and control.

\begin{tabular}{lcc}
\hline Parameter & CSC Eyes & Fellow Eyes \\
\hline Gender (male/female), n (\%) & $17(63.0) / 10(37.0)$ & $11(64.7) / 6(53.3)$ \\
Age (years) & $54(48.0 ; 63.0)$ & $54(49.5 ; 64.5)$ \\
$\begin{array}{l}\text { Disease duration, months } \\
\text { Previous treatment }\end{array}$ & $20(7 ; 48)$ & - \\
(conservative/Anti-VEGF), n (\%) & $15(55.6) / 12(44.4)$ & - \\
\hline
\end{tabular}

Data are presented as median and quartiles (Q1; Q3). CSC: Central serous chorioretinopathy; SD: standard deviation; VEGF: vascular endothelial growth factor. 
Table 2. Anatomical and functional parameters of CSC and control eyes at baseline examination.

\begin{tabular}{cccc}
\hline Parameter & CSC Eyes & Fellow Eyes & $p$-Value \\
\hline BCVA $($ logMAR $)$ & $0.4(0.1 ; 0.14)$ & $0.0(0.0 ; 0.0)$ & $<\mathbf{0 . 0 0 1}$ \\
CRT $(\mu \mathrm{m})$ & $306(254 ; 389)$ & $271(246 ; 295)$ & $\mathbf{0 . 0 4 7}$ \\
TMV $\left(\mathrm{mm}^{3}\right)$ & $8.7(8.2 ; 9.4)$ & $8,65(8.3 ; 9.08)$ & 0.697 \\
SFCT $(\mu \mathrm{m})$ & $310(286 ; 356)$ & $275(237 ; 312.8)$ & $\mathbf{0 . 0 2 2}$ \\
FRP $(\%)$ & $26.44(23.61 ; 29.52)$ & $46.77(23.49 ; 30.14)$ & 0.540 \\
CCP $(\%)$ & $43.12(38.79 ; 44.79)$ & $58.19(57.04 ; 60.36)$ & 0.159 \\
SLP $(\%)$ & $60.43(58.37 ; 63.11)$ & $61.88(58.36 ; 67.33)$ & 0.126 \\
HLP $(\%)$ & $64.27(61.24 ; 67.08)$ & $0.74(0.65 ; 0.79)$ & 0.391 \\
CCP/HLP & $0.68(0.58 ; 0.71)$ & $0.93(0.91 ; 0.99)$ & 0.142 \\
SLP/HLP & $0.95(0.92 ; 0.98)$ & 0.742 & \\
\hline
\end{tabular}

Comparison of all included CSC patients with unaffected fellow eyes at baseline examination. Mann-Whitney U test; all data are presented as median and quartiles (Q1; Q3); BCVA: best corrected visual acuity; CCP: choriocapillaris perfusion; CRT: central retinal thickness; CSC: Central serous chorioretinopathy; FRP: full retinal perfusion; HLP: Haller's layer perfusion; SFCT: subfoveal choroidal thickness; SLP: Sattler's layer perfusion; TMV: total macular volume.

Table 3. Comparison of the anatomical and functional parameters before and 4 weeks after SML for all treated patients.

\begin{tabular}{cccc}
\hline Parameter & Baseline & $\mathbf{4}$ Weeks & $p$-Value \\
\hline BCVA $(\operatorname{logMAR})$ & $0.4(0.1 ; 0.14)$ & $0.3(0.1 ; 0.5)$ & 0.348 \\
CRT $(\mu \mathrm{m})$ & $306(254 ; 389)$ & $266(236 ; 324)$ & $\mathbf{0 . 0 2 4}$ \\
TMV $\left(\mathrm{mm}^{3}\right)$ & $8.7(8.2 ; 9.4)$ & $8.5(7.9 ; 9.0)$ & 0.62 \\
SFCT $(\mu \mathrm{m})$ & $310(286 ; 356)$ & $299(273 ; 337)$ & 0.135 \\
aSRF $\left(\mu \mathrm{m}^{2}\right)$ & $0.17(0.07 ; 0.43)$ & $0.075(0.05 ; 0.33)$ & 0.115 \\
SRF area $\left(\mu \mathrm{m}^{2}\right)$ & $5.4(1.4 ; 8.25)$ & $1.8(0.7 ; 7.6)$ & 0.123 \\
LRT $(\mu \mathrm{m})$ & $364(317 ; 465)$ & $333(272 ; 391)$ & $\mathbf{0 . 0 4 4}$ \\
CCP $(\%)$ & $43.12(38.79 ; 44.79)$ & $42.35(40.97 ; 43.94)$ & 0.918 \\
SLP $(\%)$ & $60.43(58.37 ; 63.11)$ & $61.61(59.9 ; 62.81)$ & 0.469 \\
HLP $(\%)$ & $64.27(61.24 ; 67.08)$ & $63.63(60.99 ; 66.71)$ & 0.642 \\
CCP/HLP & $0.68(0.58 ; 0.71)$ & $0.65(0.61 ; 0.71)$ & 0.877 \\
SLP/HLP & $0.95(0.92 ; 0.98)$ & $0.95(0.92 ; 1.01)$ & 0.215 \\
Lesional CCP $(\%)$ & $20.45(13.58 ; 38.39)$ & $29.84(17.06 ; 35,94)$ & 0.352 \\
Lesional SLP $(\%)$ & $69.1(57.57 ; 80.39)$ & $65.33(55.95 ; 74.0)$ & 0.352 \\
Lesional HLP $(\%)$ & $72.58(57.6 ; 82.8)$ & $70.97(53.96 ; 78.83)$ & 0.352 \\
Lesional CCP/HLP & $0.29(0.17 ; 0.61)$ & $0.41(0.24 ; 0.68)$ & 0.326 \\
Lesional SLP/HLP & $0.98(0.88 ; 1.02)$ & $0.96(0.83 ; 1.15)$ & 0.959 \\
\hline
\end{tabular}

Baseline and follow-up data in all included CSC patients. Wilcoxon signed rank test; all data are presented as median and quartiles (Q1; Q3). aSRF: apical subretinal fluid; BCVA: best corrected visual acuity; CCP: choriocapillaris perfusion; CRT: central retinal thickness; CSC: Central serous chorioretinopathy; FRP: full retinal perfusion; HLP: Haller's layer perfusion; LRT: Lesional retinal thickness; SML: subthreshold microsecond pulsed laser; SFCT: subfoveal choroidal thickness; SLP: Sattler's layer perfusion; TMV: total macular volume. PNV.

Table 4 comprares demographic parameters of our subgroups: patients with CSC and

Table 5 lists anatomical and functional parameters in the two subgroups at baseline and four weeks after SML. Significant reductions were identified in the CRT of the PNV group (from $325 \mu \mathrm{m}$ to $267 \mu \mathrm{m}, p<0.01$ ) and the SFCT of the group of CSC patients without CNV (from $329 \mu \mathrm{m}$ to $299 \mu \mathrm{m}, p<0.05$ ).

Table 6 shows the statistical results of the comparison of all parameters between two subgroups, CSC without CNV and PNV, before and four weeks after treatment. The patients with PNV showed worse BCVA at both time points, but there was no difference in other parameters in OCT and OCTA. 
Table 4. Comparison of demographic parameters of patients with CSC without CNV and with CNV (PNV).

\begin{tabular}{lcc}
\hline Parameter & CSC without CNV & PNV \\
\hline Gender (male/female), n (\%) & $9(64.3) ; 5(35.7)$ & $8(61.5) ; 5(38.5)$ \\
Age (years) & $52(45.75 ; 55.75)$ & $60(52.5 ; 65)$ \\
Disease duration, months & $15(7 ; 32.5)$ & $36(9 ; 78)$ \\
\hline
\end{tabular}

Demographic data of the subgroups. Data are presented as median and quartiles (Q1; Q3). CNV: choroidal neovascularization; CSC: Central serous chorioretinopathy; PNV: pachychoroid neovasculopathy; SD: standard deviation; VEGF: vascular endothelial growth factor.

Table 5. Change in anatomical and functional parameters four weeks after SML in each subgroup.

\begin{tabular}{|c|c|c|c|c|c|c|}
\hline \multirow[t]{2}{*}{ Parameter } & \multicolumn{3}{|c|}{ CSC without CNV } & \multicolumn{3}{|c|}{ PNV } \\
\hline & Before SML & After SML & $p$-Value & Before SML & After SML & $p$-Value \\
\hline BCVA (logMAR) & $0.1(0.1 ; 0.4)$ & $0.15(0.075 ; 0.4)$ & 0.660 & $0.4(0.35 ; 0.6)$ & $0.4(0.3 ; 0.55)$ & 0.366 \\
\hline CRT $(\mu \mathrm{m})$ & $279(246 ; 385)$ & $260(245 ; 326)$ & 0.594 & $325(279 ; 411)$ & $267(211 ; 325)$ & 0.009 \\
\hline TMV & $8.45(7.9 ; 9.73)$ & 8.25 (7.88; 9.53) & 0.365 & $8.8(8.35 ; 9.15)$ & $8.6(7.95 ; 8.9)$ & 0.090 \\
\hline $\mathrm{SFCT}(\mu \mathrm{m})$ & $329(295 ; 373.75)$ & $299(284 ; 352)$ & 0.044 & $299(265 ; 332)$ & $301(272 ; 337)$ & 0.929 \\
\hline $\mathrm{aSRF}\left(\mu \mathrm{m}^{2}\right)$ & $0.12(0.06 ; 0.55)$ & $0.065(0.033 ; 0.196)$ & 0.208 & $0.26(0.09 ; 0.39)$ & $0.17(0.05 ; 0.41)$ & 0.326 \\
\hline SRF area $\left(\mu \mathrm{m}^{2}\right)$ & $4.2(1.35 ; 7.65)$ & $1.4(0.8 ; 6.33)$ & 0.208 & $5.9(1.55 ; 9.38)$ & $5.05(0.375 ; 7.75)$ & 0.406 \\
\hline LRT $(\mu \mathrm{m})$ & $370.5(310.5 ; 499.5)$ & $347(274.25 ; 405.75)$ & 0.300 & $364(310 ; 426.5)$ & $326(240.5 ; 405)$ & 0.055 \\
\hline FRP (\%) & $25.85(23.27 ; 28.39)$ & $24.88(22.75 ; 28.43)$ & 0.646 & $26.44(23.91 ; 31.02)$ & $24.91(23.37 ; 29.12)$ & 0.753 \\
\hline $\mathrm{CCP}(\%)$ & $43.39(38.32 ; 46.51)$ & $42.54(40.9 ; 44.47)$ & 0.959 & $41.92(39.26 ; 44.48)$ & $41.77(40.27 ; 44.23)$ & 0.600 \\
\hline SLP (\%) & $60.43(58.02 ; 63.16)$ & $61.88(59.66 ; 62.86)$ & 0.575 & $60.86(58.66 ; 63.57)$ & $61.22(59.99 ; 63.47)$ & 0.600 \\
\hline $\operatorname{HLP}(\%)$ & $64.19(60.66 ; 68.01)$ & $64.27(60.63 ; 67.39)$ & 0.878 & $65.02(61.28 ; 66.57)$ & $63.14(61.15 ; 65.91)$ & 0.463 \\
\hline CCP/HLP & $0.68(0.57 ; 0.74)$ & $0.66(0.61 ; 0.71)$ & 0.878 & $0.65(0.59 ; 0.71)$ & $0.66(0.60 ; 0.74)$ & 0.463 \\
\hline SLP/HLP & $0.95(0.92 ; 0.97)$ & $0.95(0.92 ; 0.98)$ & 0.575 & $0.95(0.91 ; 0.99)$ & $0.97(0.92 ; 1.0)$ & 0.075 \\
\hline Lesion CCP (\%) & $22.44(18.55 ; 42.67)$ & 30.77 (19.51; 33.97) & 0.799 & $12.86(10.11 ; 38.2)$ & $24.85(10.14 ; 49.78)$ & 0.294 \\
\hline Lesion SLP (\%) & 67.47 (56.65; 75.99) & $64.37(59.68 ; 71.06)$ & 0.445 & $74.25(55.47 ; 86.53)$ & 73.07 (48.67; 81.99) & 0.463 \\
\hline Lesion HLP (\%) & $73.39(58.46 ; 82.55)$ & $73.64(52.19 ; 81.38)$ & 0.575 & $72.58(50.66 ; 86.01)$ & $69.34(51.53 ; 80.15)$ & 0.249 \\
\hline Lesion CCP/HLP & $0.29(0.27 ; 0.71)$ & $0.41(0.32 ; 0.54)$ & 0.799 & $0.18(0.13 ; 0.65)$ & $0.46(0.13 ; 0.94)$ & 0.249 \\
\hline Lesion SLP/HLP & $0.99(0.84 ; 1.0)$ & $0.95(0.79 ; 1.11)$ & 0.799 & $0.96(0.91 ; 1.14)$ & $1.04(0.88 ; 1.27)$ & 0.917 \\
\hline
\end{tabular}

Subgroup analyses at baseline compared to the follow-up visit. Wilcoxon signed rank test, all data are presented as median and quartiles (Q1; Q3). Conservative: group of patients without prior injections or photodynamic therapy. Anti-VEGF: group of patients with prior Anti-VEGF injections; BCVA: best corrected visual acuity; CCP: choriocapillaris perfusion; CNV: choroidal neovascularization; CRT: central retinal thickness; CSC: Central serous chorioretinopathy; FRP: full retinal perfusion; HLP: Haller's layer perfusion; LRT: Lesional retinal thickness; PNV: pachychoroid neovasculopathy; SML: subthreshold microsecond pulsed laser; SFCT: subfoveal choroidal thickness; SLP: Sattler's layer perfusion; TMV: total macular volume.

Table 6. Comparison of anatomical and functional parameters of the two subgroups before and four weeks after SML.

\begin{tabular}{|c|c|c|c|c|c|c|}
\hline \multirow[t]{2}{*}{ Parameter } & \multicolumn{3}{|c|}{ Before SML } & \multicolumn{3}{|c|}{ After SML } \\
\hline & CSC without CNV & PNV & $p$-Value & CSC without CNV & PNV & $p$-Value \\
\hline BCVA (logMAR) & $0.1(0.1 ; 0.4)$ & $0.4(0.35 ; 0.6)$ & 0.007 & $0.15(0.075 ; 0.4)$ & $0.4(0.3 ; 0.55)$ & 0.014 \\
\hline CRT $(\mu \mathrm{m})$ & $278(246 ; 385)$ & $325(279 ; 411)$ & 0.259 & $260(244.75 ; 325.75)$ & $267(213 ; 325$ & 0.793 \\
\hline $\operatorname{TMV}\left(\mathrm{mm}^{3}\right)$ & $8.45(7.9 ; 9.7)$ & $8.8(8.35 ; 9.15)$ & 0.519 & $8.25(7.88 ; 9.53)$ & $8.6(7.95 ; 8.9)$ & 0.685 \\
\hline $\mathrm{SFCT}(\mu \mathrm{m})$ & $329(295 ; 373)$ & $299(265 ; 333)$ & 0.141 & $299(284 ; 352)$ & $301(273 ; 337)$ & 0.720 \\
\hline $\operatorname{aSRF}\left(\mu \mathrm{m}^{2}\right)$ & $0.12(0.06 ; 0.51)$ & $0.26(0.09 ; 0.39)$ & 0.705 & $0.07(0.033 ; 0.19)$ & 0.17 (0.05; 0.41$)$ & 0.274 \\
\hline SRF area $\left(\mu \mathrm{m}^{2}\right)$ & $4.2(1.35 ; 7.65)$ & $5.9(1.55 ; 9.38)$ & 0.689 & $1.4(0.8 ; 6.35)$ & $5.05(0.375 ; 7.57)$ & 0.852 \\
\hline LRT $(\mu \mathrm{m})$ & $371(311 ; 500)$ & $364(310 ; 427)$ & 0.981 & $347(274.25 ; 405.75)$ & $326(241 ; 405)$ & 0.583 \\
\hline FRP $(\%)$ & $25.85(23.26 ; 28.39)$ & $26.44(23.91 ; 31.02)$ & 0.635 & $24.88(22.75 ; 28.43)$ & $24.91(23.37 ; 29.12)$ & 0.792 \\
\hline CCP (\%) & $43.39(38.32 ; 46.51)$ & $41.92(39.26 ; 44.48)$ & 0.792 & $42.54(40.92 ; 44.47)$ & $41.77(40.27 ; 44.23)$ & 0.562 \\
\hline SLP (\%) & $60.43(58.02 ; 63.17)$ & $60.86(58.66 ; 63.57)$ & 0.792 & $61.89(59.66 ; 62.86)$ & $61.22(59.99 ; 63.47)$ & 0.958 \\
\hline $\operatorname{HLP}(\%)$ & $64.19(60.66 ; 68.01)$ & $65.02(61.28 ; 66.56)$ & 0.958 & $64.27(60.63 ; 67.39)$ & $63.14(61.15 ; 65.91)$ & 0.562 \\
\hline CCP/HLP & $0.68(0.57 ; 0.74)$ & $0.65(0.59 ; 0.71)$ & 0.875 & $0.66(0.61 ; 0.71)$ & $0.66(0.60 ; 0.74)$ & 0.958 \\
\hline SLP/HLP & $0.95(0.92 ; 0.99)$ & $0.95(0.92 ; 0.99)$ & 0.958 & $0.95(0.92 ; 0.98)$ & $0.97(0.92 ; 1.02)$ & 0.713 \\
\hline Lesion CCP (\%) & $22.44(18.55 ; 42.67)$ & $12.85(10.11 ; 38.20)$ & 0.118 & $30.77(19.51 ; 33.97)$ & $24.85(10.14 ; 49.78)$ & 0.635 \\
\hline Lesion SLP (\%) & $67.47(56.65 ; 75.99)$ & $74.25(55.47 ; 86.53)$ & 0.428 & $64.37(59.68 ; 71.06)$ & 73.07 (48.67; 81.99) & 0.428 \\
\hline Lesion HLP (\%) & $73.39(58.46 ; 82.55)$ & $72.58(58.46 ; 82.55)$ & 0.958 & $73.0(52.19 ; 81.38)$ & $69.34(51.53 ; 80.15)$ & 0.713 \\
\hline Lesion CCP/HLP & $0.29(0.27 ; 0.72)$ & $0.18(0.13 ; 0.65)$ & 0.220 & $0.41(0.32 ; 0.54)$ & $0.46(0.13 ; 0.95)$ & 1.0 \\
\hline Lesion SLP/HLP & $0.99(1.03 ; 0.85)$ & $0.96(0.91 ; 1.16)$ & 1.000 & $0.95(0.79 ; 1.11)$ & $1.04(0.88 ; 1.27)$ & 0.368 \\
\hline
\end{tabular}

Comparing the subgroups with CSC and PNV. Mann-Whitney U test; all data are presented as median and quartiles (Q1; Q3). aSRF: apical subretinal fluid; BCVA: best corrected visual acuity; CCP: choriocapillaris perfusion; CNV: Choroidal neovascularization; CRT: central retinal thickness; CSC: Central serous chorioretinopathy; FRP: full retinal perfusion; HLP: Haller's layer perfusion; PNV: pachychoroid neovasculopathy; SFCT: subfoveal choroidal thickness; SLP: Sattler's layer perfusion; SML: subthreshold microsecond pulsed laser; TMV: total macular volume. 


\section{Discussion}

In this prospective OCTA-based study, we investigated retinal and choroidal vascular alterations in patients diagnosed with CSC before and four weeks after SML. To the best of our knowledge, this is the first study to evaluate the impact of SML on choroidal sublayer perfusion in CSC patients in detail. Our main aim was to assess vascular changes of substructure perfusion due to SML in a short-term follow-up period. To gain deeper insights into the characteristics of conditions of the pachychoroid disease spectrum, we differentiated between CSC and PNV patients and found a different response to SML in these subgroups. The pathophysiology of CSC and other pachychoroid conditions is not fully understood, so many study groups focus on in vitro and in vivo studies.

It is known that there is an imbalance of oncotic and hydrostatic pressure in CSC due to multiple factors. Growing evidence indicates that the pathomechanisms of CSC are associated with dysfunction of the thickened choroid with subsequent impairment of the RPE. Some authors have postulated that downregulation of the cell-cell adhesion molecules in the vascular endothelium could increase the permeability of choroidal vessels, causing fluid leakage under the neurosensory retina [32]. Our study aimed to add to previous information about pathophysiological and clinical characteristics. CSC affects men more than women and usually occurs between the second and sixth life decade. Our epidemiological data concerning age and gender correspond to other studies on CSC patients [33,34]. Predictably, BCVA in our CSC cohort was significantly worse compared to unaffected eyes $(p<0.001)$, as most of the patients in this study suffered from persistent CSC with a relatively strong visual impairment and needed further intervention. Since all patients had substantial SRF before SML, CRT was significantly thicker in affected eyes than in fellow eyes $(p=0.047)$. SML has been introduced as a therapy modality for CSC with persistent fluid. For our study, we included only patients who showed SRF for at least 3 months and demonstrated typical findings of active CSC on multimodal imaging; thus, SRF would not be expected to resolve spontaneously in these patients. BCVA improved over the follow-up period ( 0.4 vs. $0.3 \log$ MAR; $p=0.348)$, even though not statistically significant. We noticed a significant reduction in CRT after SML (306 vs. $266 \mu \mathrm{m} ; p=0.024$ ). However, the BCVA and CRT outcome after SML usually change most over a longer period, which is not covered by our study design $[7,20,25]$.

Subretinal fluid parameters decreased noticeably over the follow-up period without reaching statistical significance ( $p=0.115$ for aSRF and $p=0.123$ for SRF area). The retinal thickness around the SRF lesion decreased significantly ( $364 \mathrm{vs} .333 \mu \mathrm{m} ; p=0.044$ ). It has already been shown that the morphological changes and reduction in subretinal fluid do not always correspond to the functional outcome and can be delayed [25]. However, resolution of SRF is a prerequisite for preserving or restoring function and thus the increase in the BCVA might be noticeable at later follow-up points.

As a condition of the pachychoroid disease complex, our CSC cohort had a significantly thicker SFCT than the unaffected fellow eyes $(p=0.022)$. Pachychoroid disease is characterized by the attenuation of the choriocapillaris overlying dilated choroidal veins with consecutive alteration of the RPE and retina [5]. The choroid is usually focally or diffusely thickened, which can also be seen in our measurements of the CSC group. Fellow eyes of CSC patients tend to have a thicker choroid even if they do not show any clinically significant pathologies [35]. The median choroidal thickness in our control group was $275 \mu \mathrm{m}$, which is within a normal range. However, while a thick choroid is frequently seen, choroidal thickness per se is not the most important criterion for defining the pachychoroid disease phenotype. The term pachychoroid covers several, also substructural morphological features that usually affect both eyes, even though to a different extent. It has already been reported that persistent structural changes can be detected even in unaffected fellow eyes of CSC patients [36]. Therefore, even if the unaffected fellow eyes of our control group showed no obvious pathological alterations concerning RPE and the retina, the choroid and choroidal substructures might still be altered in comparison to healthy patients. Ho et al. also investigated the changes of the choroidal thickness after SML in CSC patients [37]. 
They used a choroidal EDTRS grid to detect focal and volume changes which revealed no effect over their short-term and long-term follow-up period. They also analyzed differences in perfusion before and after SML compared to PDT in CSC patients and showed a significant change in perfusion in central $3 \times 3 \mathrm{~mm}$ angiograms of the choriocapillaris after six months but not after four weeks. We aimed to provide further detailed analyses that focus specifically on the changes in vascular sublayers to gain a deeper insight into microvascular perfusion changes. Additionally, $6 \times 6 \mathrm{~mm}$ angiograms in the current study might lead to a better overview of perfusion changes not only just around the fovea. OCTA delivers highly detailed, three-dimensional images of the microvasculature of the retina and choroid and helps to assess retinal perfusion. Several groups have already reported key findings regarding OCTA in CSC patients compared to healthy controls [38,39]. In CSC patients, the foveal architecture is distorted, and slab segmentation can be difficult. Primarily, the inner plexiform layer (IPL) is prone to inaccurate segmentation and occurs in $64.3 \%$, whereas there are almost no errors concerning segmentation of the RPE or ILM [40]. However, the IPL is the most essential layer for the correct visualization and quantification of the superficial and deep capillary plexus. To avoid the potential bias of misalignment in the inner retina, we only investigated perfusion of the full-thickness retina slab and choroidal sublayers in this study. All cases were only included after careful inspection of the segmentation.

When evaluating the choroid's sublayers at baseline, no significant differences in CSC patients compared to the fellow control eyes could be detected. FRP, CCP, SLP, and HLP did not change over the four-week follow-up when evaluating all CSC patients after SML. As CSC is a condition of the pachychoroid disease spectrum, we also analyzed the ratio in perfusion between CC, SL, and HLP. It should be noted that, even though not statistically significant, the median of the perfusion ratio CCP/HLP was lower in the CSC group compared to fellow eyes. This indicates that these sublayers of the choroid might play a role in conditions of the pachychoroid disease spectrum. It has been shown that dilated vessels, or pachyvessels are predominantly found in the Haller's layer in CSC [5] which may correspond to this finding. Over the follow-up period, perfusion values did not change significantly after SML in CSC patients. However, when looking at our subgroup analyses, we were able to detect several key findings.

Group 1 consists of CSC patients without CNV in FLA or OCTA and had only received conservative treatment modalities in the past, e.g., lifestyle changes, acetazolamide, or eplerenone. Group 2 included PNV patients, most of whom received anti-VEGF-injections in the past. None of the patients in group 1 or 2 had received PDT or conventional laser treatments before inclusion.

BCVA did not show an increase after four weeks in both groups $(p=0.66$ in group 1 ; $p=0.366$ in group 2). The median CRT decreased in both groups, but statistical significance was reached only in group 2 ( $p=0.594$ in group $1 ; p=0.009$ in group 2$)$. Interestingly, we detected a significant reduction in SFCT in the CSC group $(p=0.044)$, but not in the PNV group. The underlying mechanisms for the decrease in choroidal thickness induced by SML cannot be explained yet. Our SML protocol is adjusted to be able to irradiate RPE cells at an energy level below the threshold of cell death, which might lead to the rebuilding of the degraded RPE tight junctions, increasing pump function, and resulting in a decrease in subretinal fluid. Although it is conceivable that changes in the choroidal circulation may occur due to the effects of cytokines secreted into the basolateral direction by heated RPE cells, there is so far no basic evidence, and thus verification is necessary in future studies.

Different from the cases of CSC without CNV, the choroidal thickness of the PNV patients did not change after SML, although the retinal thickness was significantly decreased. In patients with PNV, there was no change in choroidal thickness, even though subretinal fluid was significantly reduced. Before SML, the choroidal thickness was increased in both groups, and there were no significant group differences, suggesting that CSC and PNV eyes respond differently to treatment with SML. 
Subretinal fluid was also reduced in the PNV group, suggesting that the tight junctions and pump function of the RPE may have improved through RPE hyperthermia, but the effect of laser treatment on the choroidal side may have been masked for some reason, leading to a more moderate reduction in choroidal thickness. One reason for this might be the involvement of VEGF on the choroidal side.

One limitation of the current study is the short follow-up period, even though effects are known to take several months to manifest. As described above, we aimed to gain deeper insights into short-term morphological and functional changes in chorioretinal perfusion through SML, so we focused on the results after 4 weeks. Furthermore, our treatment protocol allowed re-SML at each visit, which would have led to decreased subgroup sizes in later follow-ups. In our cohort, no one was lost to follow-up. Nevertheless, it is also necessary to investigate the effects on perfusion parameters over a longer period, such as after 3 months, 6 months, and 1 year, and for this purpose, a longer-term prospective control study is considered necessary. One of the present study's limitations is the limited number of patients; thus, the statistical power is limited, and we had difficulty identifying smaller differences between groups. Furthermore, we examined a heterogeneous group of patients (ranging from 3 to 100 months disease duration), which especially hampers the detection of clinical and functional differences in subgroups. However, for our primary endpoint, in terms of perfusion changes in OCTA before and after SML, this limitation should not play an important role.

\title{
5. Conclusions
}

In summary, our data may provide several important insights into the pathophysiology of choroidal disease and the corresponding impact of SML. The results of this study show that microsecond pulsed laser therapy may affect not only the amount of subretinal fluid but also the choroidal thickness of CSC, and that different basic pathologies respond differently to the laser therapy. These results will be of great help in determining future treatment strategies for CSC. Further research is needed to clarify the mechanism of action and long-term outcome.

Author Contributions: Conceptualization, M.P., Y.M. and M.R.; methodology, M.P., S.S. and F.R.; validation, M.R., Y.M. and S.G.; formal analysis, M.P.; investigation, A.T., V.K. and M.R.; data curation, M.P. and M.R.; writing — original draft preparation, M.P.; writing—review and editing, A.T., S.S., F.R., S.G., V.K., Y.M. and M.R.; visualization, M.P.; supervision, M.R. All authors have read and agreed to the published version of the manuscript.

Funding: This research received no external funding.

Institutional Review Board Statement: The study was conducted according to the guidelines of the Declaration of Helsinki and approved by the Ethics Committee at the University of Lübeck (protocol code 18-102, date of approval 27 June 2018).

Informed Consent Statement: Informed consent was obtained from all subjects involved in the study.

Data Availability Statement: The data presented in this study are available on request from the corresponding author. The data are not publicly available.

Conflicts of Interest: The authors declare no conflict of interest.

\author{
Abbreviations \\ aSRF apical subretinal fluid \\ BCVA best corrected visual acuity \\ CC choriocapillaris \\ CCP choriocapillaris perfusion \\ $\mathrm{CNV}$ choroidal neovascularization \\ CSC central serous chorioretinopathy \\ EDI enhanced depth of imaging
}




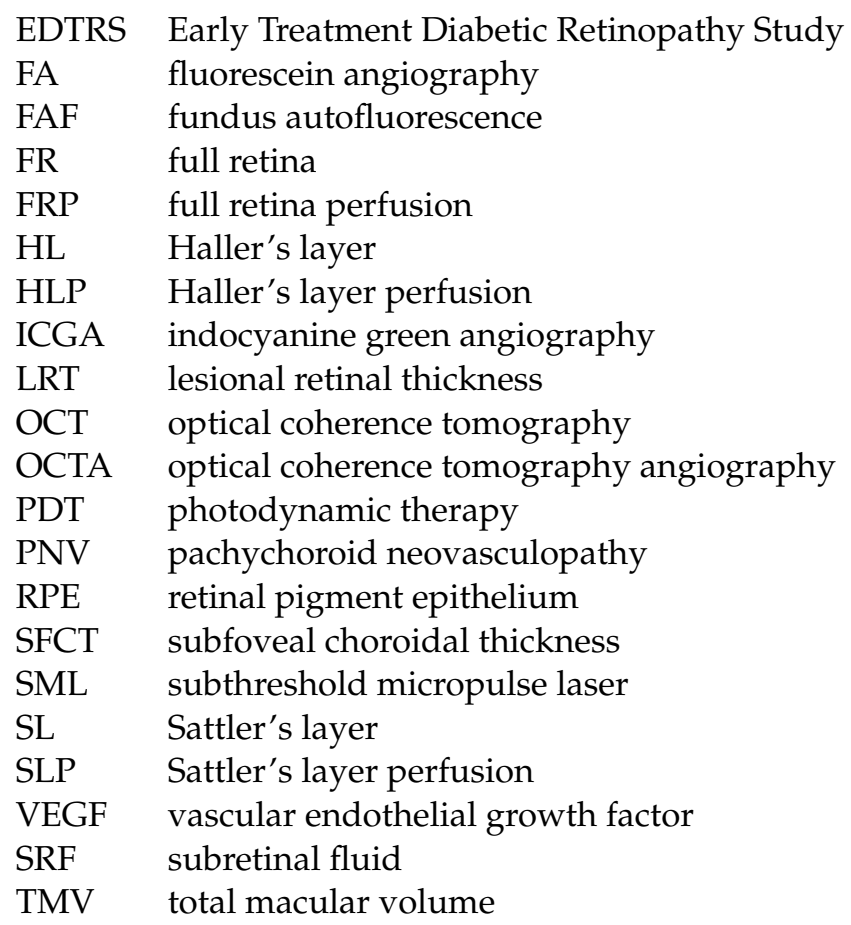

\section{References}

1. Semeraro, F.; Morescalchi, F.; Russo, A.; Gambicorti, E.; Pilotto, A.; Parmeggiani, F.; Bartollino, S.; Costagliola, C. Central Serous Chorioretinopathy: Pathogenesis and Management. Clin. Ophthalmol. Auckl. NZ 2019, 13, 2341-2352. [CrossRef]

2. Gallego-Pinazo, R.; Dolz-Marco, R.; Gómez-Ulla, F.; Mrejen, S.; Freund, K.B. Pachychoroid Diseases of the Macula. Med. Hypothesis Discov. Innov. Ophthalmol. 2014, 3, 111-115. [PubMed]

3. Warrow, D.J.; Hoang, Q.V.; Freund, K.B. Pachychoroid Pigment Epitheliopathy. Retina 2013, 33, 1659-1672. [CrossRef] [PubMed]

4. Yun, C.; Huh, J.; Ahn, S.M.; Lee, B.; Kim, J.T.; Hwang, S.-Y.; Kim, S.-W.; Oh, J. Choriocapillaris Flow Features and Choroidal Vasculature in the Fellow Eyes of Patients with Acute Central Serous Chorioretinopathy. Graefes Arch. Clin. Exp. Ophthalmol. 2019, 257, 57-70. [CrossRef]

5. $\quad$ Cheung, C.M.G.; Lee, W.K.; Koizumi, H.; Dansingani, K.; Lai, T.Y.Y.; Freund, K.B. Pachychoroid Disease. Eye Lond. Engl. 2019, 33, 14-33. [CrossRef] [PubMed]

6. Siedlecki, J.; Schworm, B.; Priglinger, S.G. The Pachychoroid Disease Spectrum-and the Need for a Uniform Classification System. Ophthalmol. Retin. 2019, 3, 1013-1015. [CrossRef]

7. Scholz, P.; Altay, L.; Fauser, S. A Review of Subthreshold Micropulse Laser for Treatment of Macular Disorders; Springer Healthcare: Cham, Switzerland, 2017; Volume 34.

8. Bousquet, E.; Dhundass, M.; Lejoyeux, R.; Shinojima, A.; Krivosic, V.; Mrejen, S.; Gaudric, A.; Tadayoni, R. Predictive Factors of Response to Mineralocorticoid Receptor Antagonists in Nonresolving Central Serous Chorioretinopathy. Am. J. Ophthalmol. 2019, 198, 80-87. [CrossRef]

9. Gallice, M.; Daruich, A.; Matet, A.; Mouvet, V.; Dirani, A.; Evequoz, G.; Geiser, M.; Behar Cohen, F.; Chiquet, C. Effect of Eplerenone on Choroidal Blood Flow Changes during Isometric Exercise in Patients with Chronic Central Serous Chorioretinopathy. Acta Ophthalmol. 2021. [CrossRef] [PubMed]

10. Sadda, S.R. Lack of Efficacy of Eplerenone for Treatment of Active Central Serous Chorioretinopathy. Eye Lond. Engl. 2020, 34, 1489-1490. [CrossRef]

11. Lotery, A.; Sivaprasad, S.; O'Connell, A.; Harris, R.A.; Culliford, L.; Cree, A.; Madhusudhan, S.; Griffiths, H.; Ellis, L.; Chakravarthy, U.; et al. Eplerenone versus Placebo for Chronic Central Serous Chorioretinopathy: The VICI RCT. In Efficacy and Mechanism Evaluation; NIHR Journals Library: Southampton, UK, 2021.

12. van Rijssen, T.J.; van Dijk, E.H.C.; Yzer, S.; Ohno-Matsui, K.; Keunen, J.E.E.; Schlingemann, R.O.; Sivaprasad, S.; Querques, G.; Downes, S.M.; Fauser, S.; et al. Central Serous Chorioretinopathy: Towards an Evidence-Based Treatment Guideline; Elsevier Ltd.: Amsterdam, The Netherlands, 2019; Volume 73.

13. Inagaki, K.; Shuo, T.; Katakura, K.; Ebihara, N.; Murakami, A.; Ohkoshi, K. Sublethal Photothermal Stimulation with a Micropulse Laser Induces Heat Shock Protein Expression in ARPE-19 Cells. J. Ophthalmol. 2015, 2015, 729792. [CrossRef]

14. Kern, K.; Mertineit, C.L.; Brinkmann, R.; Miura, Y. Expression of Heat Shock Protein 70 and Cell Death Kinetics after Different Thermal Impacts on Cultured Retinal Pigment Epithelial Cells. Exp. Eye Res. 2018, 170, 117-126. [CrossRef] [PubMed]

15. Lavinsky, D.; Wang, J.; Huie, P.; Dalal, R.; Lee, S.J.; Lee, D.Y.; Palanker, D. Nondamaging Retinal Laser Therapy: Rationale and Applications to the Macula. Investig. Ophthalmol. Vis. Sci. 2016, 57, 2488-2500. [CrossRef] [PubMed] 
16. Hirabayashi, K.; Kakihara, S.; Tanaka, M.; Shindo, T.; Murata, T. Investigation of the Therapeutic Mechanism of Subthreshold Micropulse Laser Irradiation in Retina. Graefes Arch. Clin. Exp. Ophthalmol. Albrecht Von Graefes Arch. Klin. Exp. Ophthalmol. 2020, 258, 1039-1047. [CrossRef] [PubMed]

17. Iwami, H.; Pruessner, J.; Shiraki, K.; Brinkmann, R.; Miura, Y. Protective Effect of a Laser-Induced Sub-Lethal Temperature Rise on RPE Cells from Oxidative Stress. Exp. Eye Res. 2014, 124, 37-47. [CrossRef]

18. De Cillà, S.; Vezzola, D.; Farruggio, S.; Vujosevic, S.; Clemente, N.; Raina, G.; Mary, D.; Casini, G.; Rossetti, L.; Avagliano, L.; et al. The Subthreshold Micropulse Laser Treatment of the Retina Restores the Oxidant/Antioxidant Balance and Counteracts Programmed Forms of Cell Death in the Mice Eyes. Acta Ophthalmol. 2019, 97, e559-e567. [CrossRef] [PubMed]

19. Valera-Cornejo, D.A.; García-Roa, M.; Quiroz-Mendoza, J.; Arias-Gómez, A.; Ramírez-Neria, P.; Villalpando-Gómez, Y.; RomeroMorales, V.; García-Franco, R. Micropulse Laser in Patients with Refractory and Treatment-Naïve Center-Involved Diabetic Macular Edema: Short Terms Visual and Anatomic Outcomes. Ther. Adv. Ophthalmol. 2021, 13, 2515841420979112. [CrossRef] [PubMed]

20. Uzlu, D.; Erdöl, H.; Kola, M.; Özbay, A.D. The Efficacy of Subthreshold Micropulse Yellow Laser (577 Nm) in Chronic Central Serous Chorioretinopathy. Lasers Med. Sci. 2020. [CrossRef]

21. Luttrull, J.K.; Sinclair, S.H.; Elmann, S.; Glaser, B.M. Low Incidence of Choroidal Neovascularization Following Subthreshold Diode Micropulse Laser (SDM) in High-Risk AMD. PLoS ONE 2018, 13, e0202097. [CrossRef]

22. Tode, J.; Richert, E.; Koinzer, S.; Klettner, A.; von der Burchard, C.; Brinkmann, R.; Lucius, R.; Roider, J. Thermal Stimulation of the Retina Reduces Bruch's Membrane Thickness in Age Related Macular Degeneration Mouse Models. Transl. Vis. Sci. Technol. 2018, 7, 2. [CrossRef]

23. Volodin, P.L.; Ivanova, E.V. Clinical Evaluation of Individualized and Navigated Microsecond Pulsing Laser for Acute Central Serous Chorioretinopathy. Ophthalmic Surg. Lasers Imaging Retin. 2020, 51, 512-520. [CrossRef]

24. Wu, Z.; Wang, H.; An, J. Comparison of the Efficacy and Safety of Subthreshold Micropulse Laser with Photodynamic Therapy for the Treatment of Chronic Central Serous Chorioretinopathy: A Meta-Analysis. Medicine 2021, 100, e25722. [CrossRef]

25. van Dijk, E.H.C.; Fauser, S.; Breukink, M.B.; Blanco-Garavito, R.; Groenewoud, J.M.M.; Keunen, J.E.E.; Peters, P.J.H.; Dijkman, G.; Souied, E.H.; MacLaren, R.E.; et al. Half-Dose Photodynamic Therapy versus High-Density Subthreshold Micropulse Laser Treatment in Patients with Chronic Central Serous Chorioretinopathy: The PLACE Trial. Ophthalmology 2018, 125, 1547-1555. [CrossRef]

26. Rommel, F.; Siegfried, F.; Kurz, M.; Brinkmann, M.P.; Rothe, M.; Rudolf, M.; Grisanti, S.; Ranjbar, M. Impact of Correct Anatomical Slab Segmentation on Foveal Avascular Zone Measurements by Optical Coherence Tomography Angiography in Healthy Adults. J. Curr. Ophthalmol. 2018, 30, 156-160. [CrossRef]

27. Rommel, F.; Rommel, F.; Rothe, M.; Rothe, M.; Kurz, M.; Kurz, M.; Prasuhn, M.; Prasuhn, M.; Grisanti, S.; Ranjbar, M.; et al. Evaluating Diurnal Variations in Retinal Perfusion Using Optical Coherence Tomography Angiography. Int. J. Retin. Vitr. 2020, 6. [CrossRef]

28. Siegfried, F.; Rommel, F.; Rothe, M.; Brinkmann, M.P.; Sochurek, J.A.M.; Freitag, J.; Grisanti, S.; Ranjbar, M. Evaluating Diurnal Changes in Choroidal Sublayer Perfusion Using Optical Coherence Tomography Angiography. Acta Ophthalmol. 2019, 97, e1062-e1068. [CrossRef]

29. Grading Diabetic Retinopathy from Stereoscopic Color Fundus Photographs-An Extension of the Modified Airlie House Classification. ETDRS Report Number 10. Early Treatment Diabetic Retinopathy Study Research Group. Ophthalmology 1991, 98, 786-806.

30. Otsu, N. Threshold Selection Method From Gray-Level Histograms. IEEE Trans. Syst. Man Cybern. 1979, SMC-9, 62-66. [CrossRef]

31. Nicolò, M.; Rosa, R.; Musetti, D.; Musolino, M.; Saccheggiani, M.; Traverso, C.E. Choroidal Vascular Flow Area in Central Serous Chorioretinopathy Using Swept-Source Optical Coherence Tomography Angiography. Investig. Ophthalmol. Vis. Sci. 2017, 58, 2002-2010. [CrossRef] [PubMed]

32. Sakurada, Y.; Leong, B.C.S.; Parikh, R.; Fragiotta, S.; Freund, K.B. Association between choroidal caverns and choroidal vascular hyperpermeability in eyes with pachychoroid diseases. Retina 2018, 38, 1977-1983. [CrossRef] [PubMed]

33. Daruich, A.; Matet, A.; Dirani, A.; Bousquet, E.; Zhao, M.; Farman, N.; Jaisser, F.; Behar-Cohen, F. Central Serous Chorioretinopathy: Recent Findings and New Physiopathology Hypothesis. Prog. Retin. Eye Res. 2015, 48, 82-118. [CrossRef] [PubMed]

34. Liegl, R.; Ulbig, M.W. Central Serous Chorioretinopathy. Ophthalmol. J. Int. Ophtalmol. Int. J. Ophthalmol. Z. Augenheilkd. 2014, 232, 65-76. [CrossRef] [PubMed]

35. Maruko, I.; Iida, T.; Sugano, Y.; Ojima, A.; Sekiryu, T. Subfoveal choroidal thickness in fellow eyes of patients with central serous chorioretinopathy. Retina 2011, 31, 1603-1608. [CrossRef] [PubMed]

36. Iida, T.; Kishi, S.; Hagimura, N.; Shimizu, K. Persistent and bilateral choroidal vascular abnormalities in central serous chorioretinopathy. Retina 1999, 19, 508-512. [CrossRef] [PubMed]

37. Ho, M.; Lai, F.H.P.; Ng, D.S.C.; Iu, L.P.L.; Chen, L.J.; Mak, A.C.Y.; Yip, Y.; Cheung, C.; Young, A.L.; Brelen, M. Analysis of Choriocapillaris Perfusion and Choroidal Layer Changes in Patients with Chronic Central Serous Chorioretinopathy Randomised to Micropulse Laser or Photodynamic Therapy. Br. J. Ophthalmol. 2020, 105, 555-560. [CrossRef]

38. Costanzo, E.; Cohen, S.Y.; Miere, A.; Querques, G.; Capuano, V.; Semoun, O.; El Ameen, A.; Oubraham, H.; Souied, E.H. Optical Coherence Tomography Angiography in Central Serous Chorioretinopathy. J. Ophthalmol. 2015, 2015. [CrossRef] 
39. Rochepeau, C.; Kodjikian, L.; Garcia, M.-A.; Coulon, C.; Burillon, C.; Denis, P.; Delaunay, B.; Mathis, T. Optical Coherence Tomography Angiography Quantitative Assessment of Choriocapillaris Blood Flow in Central Serous Chorioretinopathy. Am. J. Ophthalmol. 2018, 194, 26-34. [CrossRef] [PubMed]

40. Lauermann, J.L.; Woetzel, A.K.; Treder, M.; Alnawaiseh, M.; Clemens, C.R.; Eter, N.; Alten, F. Prevalences of Segmentation Errors and Motion Artifacts in OCT-Angiography Differ among Retinal Diseases. Graefes Arch. Clin. Exp. Ophthalmol. 2018, 256, 1807-1816. [CrossRef] [PubMed] 